\title{
Vandetanib-a new therapeutic option in advanced medullary thyroid cancer
}

$\mathrm{T}$ he first, large-scale, randomized phase III study in thyroid cancer provides a clear indication of efficacy for the receptor tyrosine kinase inhibitor vandetanib in patients with advanced medullary thyroid cancer (MTC). In a landmark study published in the Journal of Clinical Oncology, Wells et al. report the results that underlie the FDA's approval in April 2011 of vandetanib (also known as ZD6474) for adult patients with inoperable, metastatic or progressive MTC.

Approximately $25 \%$ of MTCs occur in a familial pattern, with the remainder arising sporadically. MTC frequently develops secondary to activating mutations in the proto-oncogene RET. Surgical removal of the thyroid is considered to be the first-line treatment for patients with MTC and its only definitive cure; however, no effective therapy exists for patients with locally advanced or metastatic disease. "On the basis of previous experimental evidence which showed that ZD6474, a smallmolecule therapeutic, inhibited oncogenic RET kinases, we conducted a phase II trial of this compound," recounts lead investigator Samuel A. Wells Jr from the National Cancer Institute, USA. "The study demonstrated that ZD6474 had therapeutic efficacy in patients with locally advanced or metastatic MTC."

Building on the phase II study, Wells and colleagues enrolled 331 patients with MTC ( $90 \%$ sporadic, $95 \%$ metastatic), aged 52 years on average, in a prospective, placebo-controlled, double-blind, randomized study, with progression-free survival determined according to the Response Evaluation Criteria in Solid Tumors (RECIST) as the primary endpoint. More than twice as many patients $(n=231)$ received vandetanib compared with those receiving placebo $(n=100)$. A crossover mechanism allowed patients who had

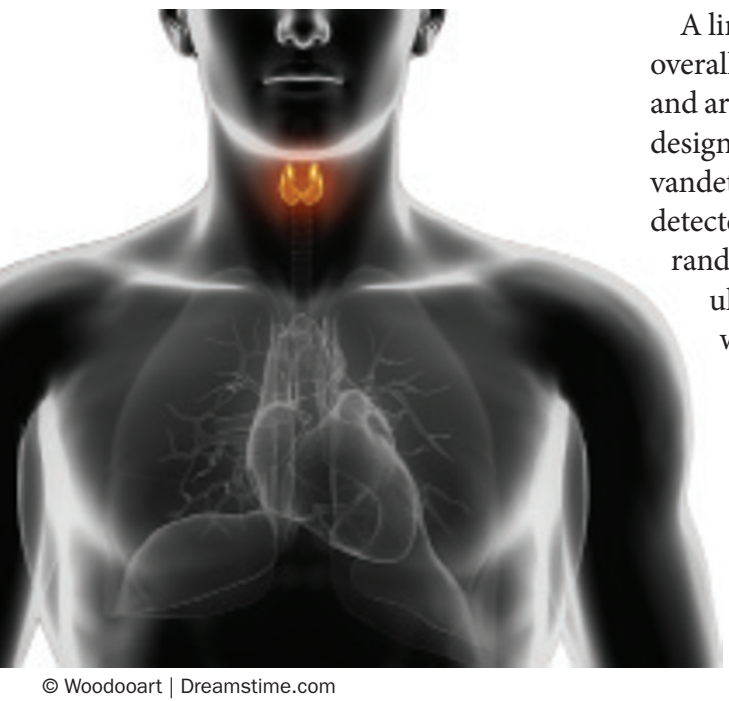

A limitation of the study is that the overall survival data have not fully matured and are likely to be compromised by a key design feature: open-label treatment with vandetanib once tumor progression is detected.Thus, a large number of patients randomly allocated to the placebo arm ultimately received vandetanib, whereas some patients allocated to vandetanib were allowed to continue taking the drug after tumor progression. "Progression-free survival is an important surrogate, but whether vandetanib and other multikinase inhibitors will produce improvements in overall survival is not yet clear," affirms Ball. Many important questions objective disease progression in the blinded phase to enter the open-label phase and receive vandetanib.

After a median follow-up period of 24 months, Wells and co-workers observed a significant improvement in the progression-free survival of patients receiving vandetanib compared with those taking placebo. A significant objective response rate (the proportion of treated patients in whom a complete or partial response is observed) was also observed for patients receiving vandetanib.

"This study is also important because it provides an important insight into the mechanism of action of the drug in patients with MTC," comments Douglas W. Ball (Johns Hopkins University School of Medicine), who was not involved in the trial. "Wells et al. showed that patients with the most common activating gene mutation in MTCs, RET Met918Thr, had better response rates and more prolonged progression-free survival than patients without this mutation." Previous studies have shown that RET Met918Thr mutation carriers are at the highest risk of recurrent disease and death among all patients with MTC. remain about the appropriate clinical use of vandetanib. Patient selection is critical, as many patients have fairly indolent disease. "Given the significant adverse effects of vandetanib and other multikinase inhibitors, including cardiac QT prolongation, a real potential for harm exists if endocrinologists and oncologists 'jump the gun' and treat patients with early or non-progressive disease," explains Ball.

Wells and co-workers now plan to conduct more clinical studies to test the efficacy of therapeutic regimens that combine a small-molecule therapeutic agent with a biologic or a standard chemotherapeutic drug. Moreover, other multikinase inhibitors that target the RET protein are in the pipeline. "Whether the suggestion of a benefit for vandetanib in patients with the tumoral Met918Thr mutation holds up will be extremely interesting to see," concludes Ball.

Linda Koch

Original article Wells, S. A. Jr et al. Vandetanib in patients
with locally advanced or metastatic medullary thyroid
cancer: a randomized, double-blind phase III trial. J. Clin.
Oncol. doi:10.1200/JC0.2011.35.5040

